\title{
Veterinary World reviewer acknowledgment 2016
}

\author{
A. V. Sherasiya and Nazir
}

Veterinary World, Star, Gulshan Park, NH-8A, Chandrapur Road, Wankaner - 363621, Dist. Morbi, Gujarat, India. Corresponding author: A. V. Sherasiya, e-mail: editorveterinaryworld@gmail.com,

Nazir: veterinaryworldpublisher@gmail.com Received: 05-01-2017, Published online: 17-01-2017

doi: 10.14202/vetworld.2017.56-60 How to cite this article: Sherasiya AV, Nazir (2017) Veterinary World reviewer acknowledgment 2016, Veterinary World, 10(1): 56-60.

\section{Contributing reviewers}

Veterinary World editorial team would sincerely like to thank all of our reviewers who contributed to peer review for the journal in 2016.

A. Aygun
Turkey

Abdulatif. A. Asheg

Libiya

\section{Abhishek Tripathi \\ USA}

Abu Nasar Md. Aminoor Rahman
Bangladesh

\section{Adel Ramoun Abd EL-Fattah}

Ramoun

Egypt

\section{Ahmed Ali Mahmoud Saleh Japan}

\section{Ahmed Mohamed Abdelaal Egypt}

\section{Ahmed Rashed Al-Najada}

South Africa

\section{Ahmed S Abdoon \\ Egypt}

\section{Ailian Geng \\ China}

\author{
Ako Faridi \\ Iran
}

\section{Alagarsamy Kannan \\ India}

\section{AliReza Bayat \\ Finland}

\section{Amarpal}

India

\author{
Amira Taman \\ Egypt
}

\section{Amlan K. Patra}

India

\section{Ammad Ahmad Farooqi \\ Pakistan}

\section{Ana BornTorrijos \\ Spain}

Ana Paula Loureiro

Brazil

\section{Anand S P}

India

Angelina Bossi Fraga
Brazil

Anna Mottola

Italy
Anna Catharina B Berge
Belgium

\section{Anna Maria Pyziel \\ Poland}

\section{Antoni Prenafeta}

Spain

\section{Antrison Morris \\ USA}
Anut Chantiratikul
Thailand

Arathy D. S. Nair

USA

\begin{abstract}
Archana Verma
India
\end{abstract}

\section{Arda Sozcu \\ Turkey}

\section{Artur Niedzwiedz \\ Poland}

\section{Arun Kumar De \\ India}

\section{Arya Sobhakumari \\ USA}

\section{Ashwani Kumar \\ India}

\section{Ayman Abdel-Aziz Swelum \\ Egypt}

Bei Wang

China

\section{Bala Manickam}

USA

Balamurugan Vinayagamurthy India

\section{Barbara Doliska \\ Poland}

\section{Beatriz \\ Spain}

Bhoj Raj Singh

India

Bijay Kumar Patra

Copyright: Sherasiya and Nazir. Open Access. This article is distributed under the terms of the Creative Commons Attribution 4.0 International License (http://creativecommons.org/licenses/by/4.0/), which permits unrestricted use, distribution, and reproduction in any medium, provided you give appropriate credit to the original author(s) and the source, provide a link to the Creative Commons license, and indicate if changes were made. The Creative Commons Public Domain Dedication waiver (http://creativecommons.org/publicdomain/zero/1.0/) applies to the data made available in this article, unless otherwise stated. 


\begin{tabular}{|c|c|c|}
\hline $\begin{array}{l}\text { Blaise Iraguha } \\
\text { Rwanda }\end{array}$ & $\begin{array}{l}\text { Archana Jain } \\
\text { India }\end{array}$ & $\begin{array}{l}\text { Giorgio Antonio Presicce } \\
\text { Italy }\end{array}$ \\
\hline $\begin{array}{l}\text { Blake J. Cochran } \\
\text { Australia }\end{array}$ & $\begin{array}{l}\text { Hari Mohan Saxena } \\
\text { India }\end{array}$ & $\begin{array}{l}\text { Giridhari Das } \\
\text { India }\end{array}$ \\
\hline $\begin{array}{l}\text { Bruce Kaplan } \\
\text { USA }\end{array}$ & $\begin{array}{l}\text { K.G. Tirumurugaan } \\
\text { India }\end{array}$ & $\begin{array}{l}\text { Giuseppe Piccone } \\
\text { Italy }\end{array}$ \\
\hline $\begin{array}{l}\text { C. Byaruhanga Byaruhanga } \\
\text { Uganda }\end{array}$ & $\begin{array}{l}\text { Lorena Mocé } \\
\text { Spain }\end{array}$ & $\begin{array}{l}\text { Gnanavel Venkatesan } \\
\text { India }\end{array}$ \\
\hline $\begin{array}{l}\text { Chandramani Pathak } \\
\text { India }\end{array}$ & $\begin{array}{l}\text { N. Punniamurthy } \\
\text { India }\end{array}$ & $\begin{array}{l}\text { Godfrey Smith } \\
\text { UK }\end{array}$ \\
\hline $\begin{array}{l}\text { Chen Yao } \\
\text { USA }\end{array}$ & $\begin{array}{l}\text { Panch Kishor Bharti } \\
\text { India }\end{array}$ & $\begin{array}{l}\text { Graciela Santillan } \\
\text { Argentina }\end{array}$ \\
\hline $\begin{array}{l}\text { Chuang Xu } \\
\text { China }\end{array}$ & $\begin{array}{l}\text { Shyamal Mondal } \\
\text { India }\end{array}$ & $\begin{array}{l}\text { Guilherme Dias de Melo } \\
\text { France }\end{array}$ \\
\hline $\begin{array}{l}\text { Chyer Kim } \\
\text { USA }\end{array}$ & $\begin{array}{l}\text { Jitendra Goswami } \\
\text { India }\end{array}$ & $\begin{array}{l}\text { Guillermo Roberto Risatti } \\
\text { USA }\end{array}$ \\
\hline $\begin{array}{l}\text { Claudia Bezerra da Silva } \\
\text { Brazil }\end{array}$ & $\begin{array}{l}\text { Pourouchottamane R. } \\
\text { India }\end{array}$ & $\begin{array}{l}\text { Gul Ahmad } \\
\text { USA }\end{array}$ \\
\hline $\begin{array}{l}\text { Danielle Bastos Araujo } \\
\text { Brazil }\end{array}$ & $\begin{array}{l}\text { Elif Çelik } \\
\text { Turkey }\end{array}$ & $\begin{array}{l}\text { Guoxiang Chao } \\
\text { China }\end{array}$ \\
\hline $\begin{array}{l}\text { David Kennedy } \\
\text { USA }\end{array}$ & $\begin{array}{l}\text { Elisabetta Manuali } \\
\text { Italy }\end{array}$ & $\begin{array}{l}\text { H. Albayrak } \\
\text { Turkey }\end{array}$ \\
\hline $\begin{array}{l}\text { Deepa P.M. } \\
\text { India }\end{array}$ & $\begin{array}{l}\text { Erdal Matur, Matur } \\
\text { Turkey }\end{array}$ & $\begin{array}{l}\text { Halit Imik } \\
\text { Turkey }\end{array}$ \\
\hline $\begin{array}{l}\text { Deepti Chachra } \\
\text { India }\end{array}$ & $\begin{array}{l}\text { Eva Voslarova } \\
\text { Czech Republic }\end{array}$ & $\begin{array}{l}\text { Hanna Markiewicz } \\
\text { Poland }\end{array}$ \\
\hline $\begin{array}{l}\text { Denisa Soledad Pérez } \\
\text { Argentina }\end{array}$ & $\begin{array}{l}\text { F. B. Peter Wooding } \\
\text { UK }\end{array}$ & $\begin{array}{l}\text { Hasan Meydan } \\
\text { Turkey }\end{array}$ \\
\hline $\begin{array}{l}\text { Denisa Soledad Pérez } \\
\text { Sudan }\end{array}$ & $\begin{array}{l}\text { Farhad Ahmadi } \\
\text { Iran }\end{array}$ & $\begin{array}{l}\text { Hassan A Hussein Hussein } \\
\text { Egypt }\end{array}$ \\
\hline $\begin{array}{l}\text { Diana Sorg } \\
\text { Germany }\end{array}$ & $\begin{array}{l}\text { Felipp Silveira Ferreira } \\
\text { Brazil }\end{array}$ & $\begin{array}{l}\text { Hazem Shaheen } \\
\text { Egypt }\end{array}$ \\
\hline $\begin{array}{l}\text { Dinh Ngoc Nguyen } \\
\text { Australia }\end{array}$ & $\begin{array}{l}\text { Filippo Giarratana } \\
\text { Italy }\end{array}$ & $\begin{array}{l}\text { Heba F Hozyen } \\
\text { Egypt }\end{array}$ \\
\hline $\begin{array}{l}\text { Doaa Mohammed Abdel Aziz } \\
\text { Egypt }\end{array}$ & $\begin{array}{l}\text { Florence D. Tarfa } \\
\text { Nigeria }\end{array}$ & $\begin{array}{l}\text { Heidrun Karlic } \\
\text { Austria }\end{array}$ \\
\hline $\begin{array}{l}\text { Domingo Fernández García } \\
\text { Spain }\end{array}$ & $\begin{array}{l}\text { Fufa Ido Gimba } \\
\text { Malaysia }\end{array}$ & $\begin{array}{l}\text { Heiner Niemann } \\
\text { Germany }\end{array}$ \\
\hline $\begin{array}{l}\text { Pradeep Kumar } \\
\text { India }\end{array}$ & $\begin{array}{l}\text { Gabriel L Cicuttin } \\
\text { Argentina }\end{array}$ & $\begin{array}{l}\text { Hilal Isleroglu } \\
\text { Turkey }\end{array}$ \\
\hline $\begin{array}{l}\text { A. K. Upadhyay } \\
\text { India }\end{array}$ & $\begin{array}{l}\text { Genevieve AndreFontaine } \\
\text { France }\end{array}$ & $\begin{array}{l}\text { Hiroshi Suzuki } \\
\text { Japan }\end{array}$ \\
\hline
\end{tabular}




\begin{tabular}{|c|c|c|}
\hline $\begin{array}{l}\text { Hui Feng } \\
\text { China }\end{array}$ & $\begin{array}{l}\text { Katarzyna Tomaszewska } \\
\text { Poland }\end{array}$ & $\begin{array}{l}\text { Marion Wassermann } \\
\text { Germany }\end{array}$ \\
\hline $\begin{array}{l}\text { Hussein Awad Hussein } \\
\text { Egypt }\end{array}$ & $\begin{array}{l}\text { Katia da Silva Calabrese } \\
\text { Brazil }\end{array}$ & $\begin{array}{l}\text { Marjana Glubovets } \\
\text { USA }\end{array}$ \\
\hline $\begin{array}{l}\text { Inga Stadaliene } \\
\text { Lithuania }\end{array}$ & $\begin{array}{l}\text { Kazuo Azuma } \\
\text { Japan }\end{array}$ & $\begin{array}{l}\text { Marta Vascellari } \\
\text { Italy }\end{array}$ \\
\hline $\begin{array}{l}\text { Ismail Hakki Tekiner } \\
\text { Turkey }\end{array}$ & $\begin{array}{l}\text { Kaïs Hussain AlGubory } \\
\text { France }\end{array}$ & $\begin{array}{l}\text { Martin M Nyaga } \\
\text { South Africa }\end{array}$ \\
\hline $\begin{array}{l}\text { Ivana Kolackova } \\
\text { Czech Republic }\end{array}$ & $\begin{array}{l}\text { Kolluri Gautham } \\
\text { India }\end{array}$ & $\begin{array}{l}\text { Martina Flöck } \\
\text { Austria }\end{array}$ \\
\hline $\begin{array}{l}\text { Jose Edmundo Apraez Guerrero } \\
\text { Colombia }\end{array}$ & $\begin{array}{l}\text { Kumari Sunita } \\
\text { India }\end{array}$ & $\begin{array}{l}\text { Mian Muhammad Awais } \\
\text { Pakistan }\end{array}$ \\
\hline $\begin{array}{l}\text { Jamal Gharekhani } \\
\text { Iran }\end{array}$ & $\begin{array}{l}\text { Kwai Lin Thong } \\
\text { Malaysia }\end{array}$ & $\begin{array}{l}\text { Miguel Lima } \\
\text { Portugal }\end{array}$ \\
\hline $\begin{array}{l}\text { James Nguhiu-Mwangi } \\
\text { Kenya }\end{array}$ & $\begin{array}{l}\text { Lia Mara Gomes Paim } \\
\text { Brazil }\end{array}$ & $\begin{array}{l}\text { Mohamed Ali Ibrahim } \\
\text { Egypt }\end{array}$ \\
\hline $\begin{array}{l}\text { Jana Jankovicova } \\
\text { Slovak }\end{array}$ & $\begin{array}{l}\text { Liben Chen } \\
\text { USA }\end{array}$ & $\begin{array}{l}\text { Mohamed Haroun } \\
\text { Qatar }\end{array}$ \\
\hline $\begin{array}{l}\text { Jeng Rung Chen } \\
\text { Taiwan }\end{array}$ & $\begin{array}{l}\text { Livia D. D. } \\
\text { Italy }\end{array}$ & $\begin{array}{l}\text { Mohie Abdallah Mahmoud } \\
\text { Haridy } \\
\text { Janan }\end{array}$ \\
\hline $\begin{array}{l}\text { Jide Owolodun } \\
\text { Nigeria }\end{array}$ & $\begin{array}{l}\text { Livio M. CostaJunior } \\
\text { Brazil }\end{array}$ & Mona Said Mahmoud \\
\hline $\begin{array}{l}\text { John P Kent } \\
\text { Ireland }\end{array}$ & $\begin{array}{l}\text { Lucia Monti } \\
\text { Italy }\end{array}$ & $\begin{array}{l}\text { Egypt } \\
\text { Moustafa kardjadj }\end{array}$ \\
\hline $\begin{array}{l}\text { Joshua Mbanga } \\
\text { Zimbabwe }\end{array}$ & $\begin{array}{l}\text { Lucie Nemcova } \\
\text { Czech Republic }\end{array}$ & Muhammad Barkaat \\
\hline $\begin{array}{l}\text { Joshua Orungo Onono } \\
\text { Kenya }\end{array}$ & $\begin{array}{l}\text { Luis Rodriguez Menocal } \\
\text { USA }\end{array}$ & $\begin{array}{l}\text { Hussain Hussain } \\
\text { Saudi Arabia }\end{array}$ \\
\hline $\begin{array}{l}\text { Joao Simoes } \\
\text { Portugal }\end{array}$ & $\begin{array}{l}\text { M AriasAlvarez } \\
\text { Spain }\end{array}$ & $\begin{array}{l}\text { Muhammad Salisu Abubakar } \\
\text { Nigeria }\end{array}$ \\
\hline $\begin{array}{l}\text { Juan Morgaz } \\
\text { Spain }\end{array}$ & $\begin{array}{l}\text { Magdalena Zajac } \\
\text { Poland }\end{array}$ & $\begin{array}{l}\text { Mustafa Cengiz } \\
\text { Turkey }\end{array}$ \\
\hline $\begin{array}{l}\text { Juliana Milani Araujo } \\
\text { Brazil }\end{array}$ & $\begin{array}{l}\text { Mahmood Habibian } \\
\text { Iran }\end{array}$ & $\begin{array}{l}\text { Mário Manuel Dinis Ginja } \\
\text { Portugal }\end{array}$ \\
\hline $\begin{array}{l}\text { Kah Hin Low } \\
\text { Malaysia }\end{array}$ & $\begin{array}{l}\text { Marcia Regina Loiko } \\
\text { Brazil }\end{array}$ & $\begin{array}{l}\text { Münir Aktas } \\
\text { Turkey }\end{array}$ \\
\hline $\begin{array}{l}\text { Kai Huang } \\
\text { USA }\end{array}$ & $\begin{array}{l}\text { Maria Emilia Eirin } \\
\text { Argentina }\end{array}$ & $\begin{array}{l}\text { Nadeem Shabir } \\
\text { India }\end{array}$ \\
\hline $\begin{array}{l}\text { Kamelia Osman } \\
\text { Egypt }\end{array}$ & $\begin{array}{l}\text { Maria-Luz Garcia } \\
\text { Spain }\end{array}$ & $\begin{array}{l}\text { Naim Deniz Ayaz } \\
\text { Turkey }\end{array}$ \\
\hline $\begin{array}{l}\text { Kang-Seuk Choi } \\
\text { Republic of Korea }\end{array}$ & $\begin{array}{l}\text { Mariette Ducatez } \\
\text { France }\end{array}$ & $\begin{array}{l}\text { Natalia Kasica } \\
\text { Poland }\end{array}$ \\
\hline
\end{tabular}




\begin{tabular}{|c|c|c|}
\hline $\begin{array}{l}\text { Nazli Ercan } \\
\text { Turkey }\end{array}$ & $\begin{array}{l}\text { Rafael Cardoso Carvalho } \\
\text { Brazil }\end{array}$ & $\begin{array}{l}\text { Sebastian Nowaczewski } \\
\text { Poland }\end{array}$ \\
\hline $\begin{array}{l}\text { Nicholas Johnson } \\
\text { UK }\end{array}$ & $\begin{array}{l}\text { Rajesh Somasundaram } \\
\text { Sweden }\end{array}$ & $\begin{array}{l}\text { Selwyn Arlington Headley } \\
\text { Brazil }\end{array}$ \\
\hline $\begin{array}{l}\text { Nikolaos D Soultos } \\
\text { Greece }\end{array}$ & $\begin{array}{l}\text { Rajib Deb } \\
\text { India }\end{array}$ & $\begin{array}{l}\text { Serena Montagnaro } \\
\text { Italy }\end{array}$ \\
\hline $\begin{array}{l}\text { Nuh Kilic } \\
\text { Turkey }\end{array}$ & $\begin{array}{l}\text { Ramesh Dharmaraj } \\
\text { India }\end{array}$ & $\begin{array}{l}\text { Serpil Sariozkan } \\
\text { Turkey }\end{array}$ \\
\hline $\begin{array}{l}\text { Nuriye Ezgi Bektur } \\
\text { Turkey }\end{array}$ & $\begin{array}{l}\text { Raquel Salazar Lugo } \\
\text { Venezuela }\end{array}$ & $\begin{array}{l}\text { Shahzad Ali } \\
\text { Pakistan }\end{array}$ \\
\hline $\begin{array}{l}\text { Olga Zorman Rojs } \\
\text { Slovenia }\end{array}$ & $\begin{array}{l}\text { Rasheed Adetola Ajadi } \\
\text { Nigeria }\end{array}$ & $\begin{array}{l}\text { Shailbala Singh } \\
\text { USA }\end{array}$ \\
\hline $\begin{array}{l}\text { Ovais Aarif } \\
\text { India }\end{array}$ & $\begin{array}{l}\text { Ratna Ghosal } \\
\text { USA }\end{array}$ & $\begin{array}{l}\text { Shiro Murata } \\
\text { Japan }\end{array}$ \\
\hline $\begin{array}{l}\text { Pabitra Hriday Patra } \\
\text { UK }\end{array}$ & $\begin{array}{l}\text { Robert Gilbert } \\
\text { USA }\end{array}$ & $\begin{array}{l}\text { Shivaramu Keelara } \\
\text { Veerappa } \\
\text { USA }\end{array}$ \\
\hline Preece & Rodolfo Ungerfeld & $\begin{array}{l}\text { Shoja Jafari } \\
\text { Iran }\end{array}$ \\
\hline India & Uruguay & $\begin{array}{l}\text { Silvana Scarcella } \\
\text { Argentina }\end{array}$ \\
\hline $\begin{array}{l}\text { India } \\
\text { Partha Ray }\end{array}$ & $\begin{array}{l}\text { Ebensperger } \\
\text { Spain }\end{array}$ & $\begin{array}{l}\text { Sinan Ince } \\
\text { Turkey }\end{array}$ \\
\hline $\begin{array}{l}\text { USA } \\
\text { Paul J. Brindley }\end{array}$ & $\begin{array}{l}\text { Rosa María Garcia Garcia } \\
\text { Spain }\end{array}$ & $\begin{array}{l}\text { Socorro RetanaMarquez } \\
\text { Mexico }\end{array}$ \\
\hline $\begin{array}{l}\text { USA } \\
\text { Piyumali K Perera }\end{array}$ & $\begin{array}{l}\text { Rute M Noiva } \\
\text { Portugal }\end{array}$ & $\begin{array}{l}\text { Sohair A M Eltony } \\
\text { Egypt }\end{array}$ \\
\hline Sri Lanka & $\begin{array}{l}\text { S L Choubisa } \\
\text { India }\end{array}$ & $\begin{array}{l}\text { Somia H AbdAllah } \\
\text { Egypt }\end{array}$ \\
\hline Iran & $\begin{array}{l}\text { S.K. Mondal } \\
\text { India }\end{array}$ & $\begin{array}{l}\text { Somwang Lekjing } \\
\text { Thailand }\end{array}$ \\
\hline $\begin{array}{l}\text { India } \\
\text { Prem Singh Yadav }\end{array}$ & $\begin{array}{l}\text { Sagar Sanyal } \\
\text { India }\end{array}$ & $\begin{array}{l}\text { Sorin Daniel Dan } \\
\text { Romania }\end{array}$ \\
\hline India & $\begin{array}{l}\text { Sameer J Mabjeesh } \\
\text { Israel }\end{array}$ & $\begin{array}{l}\text { Stefania Perrucci } \\
\text { Italy }\end{array}$ \\
\hline China & $\begin{array}{l}\text { Sana A M Elgayar } \\
\text { Egypt }\end{array}$ & $\begin{array}{l}\text { Sudson Sirivaidyapong } \\
\text { Thailand }\end{array}$ \\
\hline China & $\begin{array}{l}\text { Sandeep Bhatia } \\
\text { India }\end{array}$ & $\begin{array}{l}\text { Sunil More } \\
\text { USA }\end{array}$ \\
\hline USA & $\begin{array}{l}\text { Sandeep Ghatak } \\
\text { Inda }\end{array}$ & $\begin{array}{l}\text { Sushil Kumar maiti } \\
\text { India }\end{array}$ \\
\hline $\begin{array}{l}\text { Cosendey KezenLeite } \\
\text { Brazil }\end{array}$ & $\begin{array}{l}\text { Sasimanas Unajak } \\
\text { Thailand }\end{array}$ & $\begin{array}{l}\text { Svjetlana Terzic } \\
\text { Croatia }\end{array}$ \\
\hline
\end{tabular}




\begin{tabular}{lll}
\hline Takuya Aikawa & Vikas Vohra & Ya-Ching Lin \\
Japan & India & Taiwan \\
Tanko Polycarp Nwunuji & Vincenzo Tufarrelli & Ya-Ling Huang \\
Nigeria & Italy & Taiwan \\
Teodulo Quezada Tristan & Vishal S Suthar & Yan-Chen Bo \\
Mexico & India & China \\
Thomas J Nolan & W Senosy & Yashpal Singh Malik \\
USA & Egypt & India \\
Tomasz Suchocki & Walied Sobhy Abdo & Yasmina Mohammed \\
Poland & Egypt & Abd El Hakim El Ghazaly \\
Tulin Guven Gokmen & Wenliang Li & Egypt \\
Turkey & China & Yassir Adam Shuaib \\
Uma Kant Mishra & William Alberto Canon Franco & Sudan \\
India & Colombia & Zahid Naseer \\
Umut Tasdemir & Woo-Jin Jung & Pakistan \\
Turkey & Republic of Korea & Zhuo-Ming Qin \\
V Pirgozliev & Xing Ping Wang & China \\
UK & China & Zuhair Ismail \\
Vijaya Kumar Anumolu & Yavuz Gurbuz & Jordan \\
India & Turkey & \\
& & * $* * * * * * *$
\end{tabular}

